\title{
A new approach of superconvergence analysis of a low order nonconforming MFEM for reaction-diffusion equation
}

Houchao Zhang ${ }^{1 *}$ and An Wang ${ }^{1}$

"Correspondence:

zhc0375@126.com

'School of Mathematics and

Statistics, Pingdingshan University,

Pingdingshan, P.R. China

\begin{abstract}
In this paper, a low order nonconforming mixed finite element method (MFEM) is studied with $E Q_{1}^{\text {rot }}$ element and zero order Raviart-Thomas (R-T) element for a class of nonlinear reaction-diffusion equations. On the one hand, a priori bound is proved using Lyapunov functional, which leads to the global existence and uniqueness of the approximation solutions. Further, the superclose estimates of order $O\left(h^{2}\right)$ for original variable $u$ in broken $H^{1}$ norm and the flux $\boldsymbol{p}=\nabla u$ in $\left(L^{2}\right)^{2}$ norm are obtained respectively for a semi-discrete scheme. On the other hand, a linearized Crank-Nicolson fully-discrete scheme is established and the superclose estimates of order $O\left(h^{2}+\tau^{2}\right)$ are also obtained unconditionally by making full use of the special characters of the above mixed finite elements (MFEs) and two different splitting techniques, which are used to deal with the consistency errors and nonlinear terms, respectively. These approaches circumvent the restrictive condition on a time step size arising as an inverse inequality used to prove a posteriori bounds in $L^{\infty}$ norm, which is necessary for nonlinear problems for the conventional finite element analysis. What is more, the corresponding global superconvergent results are derived through interpolated postprocessing techniques. Finally, numerical results are provided to confirm the theoretical analysis. Here $h$ is the subdivision parameter and $\tau$ is the time step.
\end{abstract}

Keywords: Reaction-diffusion equation; Mixed finite element method; Linearized fully discrete scheme; Superconvergence

\section{Introduction}

Consider the following nonlinear reaction-diffusion equation:

$$
\begin{cases}u_{t}-\Delta u+f(u)=0, & (\boldsymbol{x}, t) \in \Omega \times(0, T], \\ u(\boldsymbol{x}, t)=0, & (\boldsymbol{x}, t) \in \partial \Omega \times(0, T], \\ u(\boldsymbol{x}, 0)=u_{0}, & \mathbf{x} \in \Omega,\end{cases}
$$

where $\boldsymbol{x}=(x, y), 0<T<\infty$, and $\Omega \in \mathbb{R}^{2}$ is a rectangle with the boundary $\partial \Omega$. $u_{0}$ is a given function. Equation (1) arises in cell dynamics behavior [1], ecological invasion [2], chemical reaction modeling [3], and so forth. The mathematical nature of Equation (1) is determined by a kinetic function $f(\cdot)$, and its analytical properties were widely investigated

(c) The Author(s) 2018. This article is distributed under the terms of the Creative Commons Attribution 4.0 International License (http://creativecommons.org/licenses/by/4.0/), which permits unrestricted use, distribution, and reproduction in any medium, provided you give appropriate credit to the original author(s) and the source, provide a link to the Creative Commons license, and indicate if changes were made. 
by many researchers. For example, the case $f(u)=u-u^{2}$ was studied initially by Fisher (1937) and Kolmogorov et al. (1937) [4], the case $f(u)=u(u-\alpha)(1-u), \alpha \in(0,1)$ was studied by Fife and McLeod (1977) [5], and the case $f(u)=u^{3}-u$ was studied by Sherratt and Marchant (1996) [6]. However, it is quite difficult to construct the exact solutions for partial differential equations (PDEs). This restraints practical applications of PDEs. Therefore, the numerical methods for solving PDEs are highly desired. In this work, we focus on MFEM with $E Q_{1}^{\text {rot }}$ element and zero order Raviart-Thomas $(\mathrm{R}-\mathrm{T})$ element for nonlinear reaction-diffusion equation (1) with reaction term $f(u)=u^{3}-u$.

In the past several decades, numerous efforts have been devoted to the development of efficient numerical methods for solving the reaction-diffusion equations. For example, [7-10] presented finite difference methods in one and multi-dimensions. [11, 12] studied finite element methods. Since the pioneering work of Raviart and Thomas [13], MFEMs have had many attractive features over the conventional Lagrange FEMs. For instance, MFEMs conserve mass locally, which is of crucial importance in numerical methods for flow coupled to transport [14]. By introducing $\nabla u$ as an extra variable, mixed methods can produce accurate flux approximations. There are numerous applications of MFEMs for general linear and nonlinear reaction-diffusion equations, see [15-21]. A two-grid method for expanded MFEMs of semilinear reaction-diffusion equations was investigated by $[22,23]$.

To the best of our knowledge, the above interesting works analyzed the optimal error estimates for conforming (mixed) finite elements, and the function $f(u)$ requires different conditions. For example, the function $f(u)$ is a smooth function of $u \in \mathbb{R}$ in [20], $|f| \leq k$, and $\left|\frac{\partial f}{\partial u}\right| \leq k$ ( $k$ is a positive constant) in $[16,17], f$ is twice continuously differential on $\Omega$ with bounded derivatives up to the second order in [21], and $f$ is a triple continuously differential function with bounded derivatives up to the third order in $[15,22,23]$. All these strong assumptions on $f$ make nonlinear equation (1) almost a linear one and the error between $f(u)$ and $f\left(u_{h}\right)$ can be bounded by

$$
\left\|f(u)-f\left(u_{h}\right)\right\|_{0}=\left\|f^{\prime}\left(\mu_{u, u_{h}}\right)\left(u-u_{h}\right)\right\|_{0} \leq \max _{\boldsymbol{x} \in \mathbf{R}}\left|f^{\prime}(\boldsymbol{x})\right|\left\|u-u_{h}\right\|_{0} \leq L \mid\left\|u-u_{h}\right\|_{0},
$$

where $L$ is a positive constant independent of $u, u_{h}$ and the mesh size $h\left(u_{h}\right.$ stands for numerical solution). Clearly, these assumptions on $f$ cannot be satisfied in most applications. For instance, $f(u)=u^{3}-u$ is frequently used in phase field problems and nonlinear Schrödinger equations. Thus, all the previous results are not applicable. To eliminate the strong assumptions of $f$, one must derive a uniform boundedness of $u_{h}$ in certain strong norms. A so-called a posteriori bound in $L^{\infty}$ norm was used to derive optimal error estimates of conventional (mixed) FEMs, time step size restrictions were also required to circumvent the difficulties caused by nonlinear term $f(u)$ [24]. The time step restrictions may lead to the use of an unnecessarily small time step and make the computations much more time-consuming. In order to avoid such defects, the so-called splitting technique was introduced in $[25,26]$ and was later used in [27-35], where a corresponding timediscrete system was constructed to split the error into two parts, the temporal error and the spatial error.

In this paper, we may develop another approach to study the unconditional superconvergence analysis for Equation (1) with a new low order nonconforming MFEM introduced 
in [36, 37], which is different from the conventional MFEM used in [13, 15-21, 38]. To circumvent the difficulties caused by nonlinear terms $f(u)$ and to relax the restrictions of $f(u)$, we use a novel splitting technique to split the nonlinear term $f(u)$ into different parts, use the $\|\cdot\|_{0,2 p}$ norm to estimate the splitting terms. Through a sharp estimate, we derive the desired results. On the other hand, there exists the consistency error for nonconforming elements, which also leads to losing error order and causes time step restrictions. For example, $E Q_{1}^{\text {rot }}$ nonconforming $\mathrm{FE}$, the consistency error of this element is only estimated as follows:

$$
\sum_{K} \int_{\partial K} \frac{\partial u}{\partial \boldsymbol{n}} \varphi_{h} d s \leq C h^{2}|u|_{3}\left\|\varphi_{h}\right\|_{1, h} \leq C h|u|_{3}\left\|\varphi_{h}\right\|_{0} .
$$

Only order $O(h)$ result can be deduced ( $\varphi_{h}$ belongs to $E Q_{1}^{\text {rot }}$ finite element space). Therefore, another novel splitting argument for consistency error is also developed, by which the consistency errors are split into different parts and the time steps are transferred for one part of the integral to another. This technique also removes time step restrictions.

The paper is organized as follows. In Sect. 2, a brief description of nonconforming MFEs is introduced and some useful high accuracy results are given. In Sect. 3, a priori bound is proved using Lyapunov functional, which leads to the global existence and uniqueness of the approximation solutions. Further, the superclose estimates of order $O\left(h^{2}\right)$ for original variable $u$ in broken $H^{1}$ norm and the flux $\boldsymbol{p}=\nabla u$ in $\left(L^{2}\right)^{2}$ norm are obtained respectively for a semi-discrete scheme. In Sect. 4, a linearized Crank-Nicolson fully-discrete scheme is established and the superclose estimates of order $O\left(h^{2}+\tau^{2}\right)$ are obtained. In Sect. 5, the superconvergence estimates are derived through interpolated postprocessing techniques. In Sect. 6, some numerical results are provided to show the validity of the theoretical analysis. Finally, conclusions and discussions are summarized in Sect. 7.

\section{Construction of mixed finite elements}

Let $\Omega \subset \mathbb{R}^{2}$ be a convex polygon with boundary $\partial \Omega$ parallel to the coordinate axes, $\Gamma_{h}$ be a rectangular subdivision. For any given $K \in \Gamma_{h}$, let the four vertices and edges be $A_{i}(x, y)$ $(i=1,2,3,4 \cdot \bmod (4))$ and $l_{i}=\overline{A_{i} A_{i+1}}(i=1,2,3,4 \cdot \bmod (4))$, respectively. The MFE spaces $M_{h}$ and $\boldsymbol{W}_{h}$ are taken as [39]:

$$
\begin{aligned}
& M_{h}=\left\{\varphi_{h} ;\left.\varphi_{h}\right|_{K} \in \operatorname{span}\left\{1, x, y, x^{2}, y^{2}\right\}, \int_{\mathcal{F}}\left[\varphi_{h}\right] d s=0, F \subset \partial K, \forall K \in \Gamma_{h}\right\}, \\
& \boldsymbol{W}_{h}=\left\{\boldsymbol{w}_{h}=\left(w_{h}^{1}, w_{h}^{2}\right) ;\left.\boldsymbol{w}_{h}\right|_{K} \in Q_{1,0}(K) \times Q_{0,1}(K), \forall K \in \Gamma_{h}\right\},
\end{aligned}
$$

where $Q_{i j}=\operatorname{span}\left\{x^{r} y^{s}, 0 \leq r \leq i, 0 \leq s \leq j\right\}$. [ $\left.\varphi_{h}\right]$ stands for jump of $\varphi_{h}$ across the edge $\mathcal{F}$ if $\mathcal{F}$ is an internal edge, and $\left[\varphi_{h}\right]=\varphi_{h}$ if $\mathcal{F}$ is a boundary edge.

Let $I_{h}: u \in H^{1}(\Omega) \rightarrow I_{h} u \in M_{h}$ and $\Pi_{h}=\left(\Pi_{h}^{1}, \Pi_{h}^{2}\right): \boldsymbol{w}=\left(w^{1}, w^{2}\right) \in\left(H^{1}(\Omega)\right)^{2} \rightarrow \Pi_{h} \boldsymbol{w}=$ $\left(\Pi_{h}^{1} w^{1}, \Pi_{h}^{2} w^{2}\right) \in \boldsymbol{W}_{h}$ be the associated interpolation operators on $M_{h}$ and $\boldsymbol{W}_{h}$, respectively, which satisfy $\left.I_{h}\right|_{K}=I_{K},\left.\Pi_{h}\right|_{K}=\left(\left.\Pi_{h}^{1}\right|_{K},\left.\Pi_{h}^{2}\right|_{K}\right)=\Pi_{K}=\left(\left.\Pi^{1}\right|_{K},\left.\Pi^{2}\right|_{K}\right)$,

$$
\int_{K}\left(u-I_{K} u\right) d \mathbf{x}=0, \quad \int_{l_{i}}\left(u-I_{K} u\right) d s=0, \quad i=1,2,3,4,
$$


and

$$
\int_{l_{i}}\left(\boldsymbol{w}-\Pi_{K} \boldsymbol{w}\right) \cdot \boldsymbol{n}_{i} d s=0, \quad i=1,2,3,4,
$$

where $\boldsymbol{n}_{i}$ is the unit outward normal vector to boundary $l_{i}(i=1,2,3,4)$.

The following results play an important role in the forgoing superconvergence analysis. Equations (2), (3), and (4) can be found in [40, 41], and [42], respectively.

For $u \in H^{3}(\Omega), \varphi_{h} \in M_{h}, \boldsymbol{w}_{h} \in \boldsymbol{W}_{h}$, there hold

$$
\begin{aligned}
& \left(\boldsymbol{p}-\Pi_{h} \boldsymbol{p}, \boldsymbol{w}_{h}\right) \leq C h^{2}|\boldsymbol{p}|_{2}\left\|\boldsymbol{w}_{h}\right\|_{0}, \\
& \left(\nabla_{h}\left(u-I_{h} u\right), \nabla_{h} \varphi_{h}\right)_{h}=0,
\end{aligned}
$$

and

$$
\sum_{K} \int_{\partial K} \boldsymbol{p} \cdot \boldsymbol{n} \varphi_{h} d s \leq C h^{2}|\boldsymbol{p}|_{2}\left\|\varphi_{h}\right\|_{1, h},
$$

where $\nabla_{h}$ denotes the gradient operator defined piecewisely, $(u, v)_{h}=\sum_{K} \int_{K} u v d x d y$, $\|\cdot\|_{1, h}=\left(\sum_{K}|\cdot|_{1, K}\right)^{1 / 2}$ is a norm on $M_{h}$. C denotes a generic positive constant, which is independent of $h, \tau$, but may depend on $u$ and $f$.

\section{Superclose analysis for a semi-discrete scheme}

In this section, we will present a spatial discrete scheme for Equation (1), discuss the existence, uniqueness, stability of the approximate solution $u_{h}$, and derive the superclose estimates.

Let $\boldsymbol{p}=-\nabla u$, then Equation (1) can be put in the form

$$
\begin{cases}u_{t}+\nabla \cdot \boldsymbol{p}+f(u)=0, & (\boldsymbol{x}, t) \in \Omega \times(0, T], \\ \boldsymbol{p}+\nabla u=0, & (\boldsymbol{x}, t) \in \Omega \times(0, T], \\ u(\boldsymbol{x}, t)=0, & (\boldsymbol{x}, t) \in \partial \Omega \times(0, T], \\ u(\boldsymbol{x}, 0)=u_{0}, & \boldsymbol{x} \in \Omega,\end{cases}
$$

and the corresponding weak formulation is to find $\{u, \boldsymbol{p}\}:(0, T] \rightarrow H_{0}^{1}(\Omega) \times\left(L^{2}(\Omega)\right)^{2}$ such that

$$
\begin{cases}\left(u_{t}, \varphi\right)-(\boldsymbol{p}, \nabla \varphi)+(f(u), \varphi)=0, & \forall \varphi \in H_{0}^{1}(\Omega), \\ (\boldsymbol{p}, \boldsymbol{w})+(\nabla u, \boldsymbol{w})=0, & \forall \boldsymbol{w} \in\left(L^{2}(\Omega)\right)^{2}, \\ u(\boldsymbol{x}, 0)=u_{0}, & \boldsymbol{x} \in \Omega .\end{cases}
$$

Consider the approximation of (5): to seek $\left\{u_{h}, \boldsymbol{p}_{h}\right\} \in M_{h} \times \boldsymbol{W}_{h}$ such that

$$
\begin{cases}\left(u_{h t}, \varphi_{h}\right)-\left(\boldsymbol{p}_{h}, \nabla_{h} \varphi_{h}\right)_{h}+\left(f\left(u_{h}\right), \varphi_{h}\right)=0, & \forall \varphi_{h} \in M_{h}, \\ \left(\boldsymbol{p}_{h}, \vec{w}_{h}\right)+\left(\nabla_{h} u_{h}, \boldsymbol{w}_{h}\right)_{h}=0, & \forall \boldsymbol{w}_{h} \in \boldsymbol{W}_{h}, \\ u_{h}(\boldsymbol{x}, 0)=I_{h} u_{0}, & \boldsymbol{x} \in \Omega .\end{cases}
$$


Remark 1 Compared with the classical mixed finite element approximation schemes studied by $[13,15-21,38]$, the gradient in our scheme belongs to the simple square integrable space $\left(L^{2}(\Omega)\right)^{2}$ avoiding the use of the complex space $\boldsymbol{H}(\operatorname{div} ; \Omega)$. Obviously, the regularity required on the solution $\boldsymbol{p}=-\nabla u$ is reduced.

Theorem 1 Problems (6) are uniquely solvable and there holds

$$
\left\|u_{h}\right\|_{1, h} \leq C .
$$

Proof In terms of the bases $\left\{\varphi_{i}\right\}_{i=1}^{r_{1}}$ for $M_{h}$ and $\left\{\boldsymbol{\psi}_{j}\right\}_{j=1}^{r_{2}}$ for $\boldsymbol{W}_{h}$, we can suppose that

$$
u_{h}=\sum_{i=1}^{r_{1}} u_{i}(t) \varphi_{i}, \quad \boldsymbol{p}_{h}=\sum_{j=1}^{r_{2}} p_{j}(t) \boldsymbol{\psi}_{j}
$$

Then problem (6) can be written as follows: Find $u_{i}(t)$ and $p_{j}(t), i=1,2, \ldots, r_{1}, j=$ $1,2, \ldots, r_{2}$, such that, $\forall t \in[0, t]$,

$$
\left\{\begin{array}{l}
\text { (a) } \quad \boldsymbol{A} \frac{d \boldsymbol{U}(t)}{d t}-\boldsymbol{B P}(t)+\boldsymbol{F}(\boldsymbol{U}(t))=\mathbf{O} \\
\text { (b) } \boldsymbol{C P}(t)+\boldsymbol{B}^{\prime} \boldsymbol{U}(t)=0 \\
\text { (c) } \boldsymbol{U}(0)=\boldsymbol{U}_{0}
\end{array}\right.
$$

where

$$
\begin{aligned}
& \boldsymbol{U}(t)=\left(u_{1}(t), u_{2}(t), \ldots, u_{r_{1}}(t)\right)^{\prime}, \quad \boldsymbol{P}(t)=\left(p_{1}(t), p_{2}(t), \ldots, p_{r_{2}}(t)\right)^{\prime}, \\
& \boldsymbol{U}_{0}=\left(u_{1}(0), u_{2}(0), \ldots, u_{r_{1}}(0)\right)^{\prime}, \quad \boldsymbol{A}=\left(\left(\varphi_{i}, \varphi_{j}\right)\right)_{r_{1} \times r_{1}}, \\
& \boldsymbol{B}=\left(\left(\nabla_{h} \varphi_{i}, \boldsymbol{\psi}_{j}\right)_{h}\right)_{r_{1} \times r_{2}}, \quad \boldsymbol{C}=\left(\left(\boldsymbol{\psi}_{i}, \boldsymbol{\psi}_{j}\right)\right)_{r_{2} \times r_{2}}, \\
& \boldsymbol{F}(\boldsymbol{U})=\left(\left(f\left(\sum_{k=1}^{r_{1}} u_{k}(t) \varphi_{k}\right), \varphi_{i}\right)\right)_{r_{1} \times 1} .
\end{aligned}
$$

Since the matrices $\boldsymbol{A}, \boldsymbol{C}$ are positive definite, we can derive from (8(b))

$$
\boldsymbol{P}(t)=-\boldsymbol{C}^{-1} \boldsymbol{B}^{\prime} \boldsymbol{U}(t) .
$$

Substituting (9) into (8(a)), we get

$$
\left\{\begin{array}{l}
\boldsymbol{A} \frac{d \boldsymbol{U}(t)}{d t}+\boldsymbol{B C}^{-1} \boldsymbol{B}^{\prime} \boldsymbol{U}(t)+\boldsymbol{F}(\boldsymbol{U}(t))=\mathbf{O} \\
\boldsymbol{U}(0)=\boldsymbol{U}_{0} .
\end{array}\right.
$$

Clearly, (10) presents nonlinear ordinary differential equations with locally Lipschitz continuity on $\boldsymbol{F}$. The Picard theorem ensures that system (10) admits a unique local solution $\boldsymbol{U}(t)$ on a certain maximal subinterval $\left(0, t_{h}\right]$ of $[0, T]$. For proving the global existence, we need a priori bounds to continue beyond $t_{h}$. To do this, we introduce a new Lyapunov functional $L(\varphi)$ :

$$
L(\varphi)=\frac{1}{2}\left(\nabla_{h} \varphi, \nabla_{h} \varphi\right)_{h}+(F(\varphi), 1) .
$$


Moreover, taking $\left\{v_{h}, \boldsymbol{w}_{h}\right\}=\left\{u_{h t}, \nabla_{h} u_{h t}\right\}$ in (6) and adding the first two equations, we have

$$
\frac{d L\left(u_{h}\right)}{d t}=-\left\|u_{h t}\right\|_{0}^{2} \leq 0
$$

Integrating both sides with respect to time variable from 0 to $t$, we have

$$
L\left(u_{h}\right) \leq L\left(u_{h}(0)\right)
$$

which implies that

$$
\left\|\nabla_{h} u_{h}\right\|_{0} \leq \sqrt{\left\|\nabla_{h} u_{h}(\boldsymbol{x}, 0)\right\|_{0}^{2}+2\left(F\left(u_{h}(\boldsymbol{x}, 0)\right), 1\right)} \leq C .
$$

Thus, we conclude the global existence of a solution $\boldsymbol{U}(t)$ to (10). Then we can also get $\boldsymbol{P}(t)$ from (9). In other words, problem (6) is uniquely solvable.

Remark 2 The key to the proof of (7) is the construction of a Lyapunov functional $L(\varphi)$, which is not an easy thing for the general function $f(\cdot)$, and allows us to get rid of the assumption on boundedness of numerical solution $\left\|u_{h}\right\|_{0, \infty}$, which is usually necessary and inevitable in the traditional finite element analysis.

In this paper, the following inequality will be always used [43]:

$$
\left\|\varphi_{h}\right\|_{0,2 p} \leq C\left\|\varphi_{h}\right\|_{1, h}, \quad \forall \varphi_{h} \in M_{h}, p \in \mathbb{N}^{+} .
$$

Theorem 2 Let $\{u, \boldsymbol{p}\}$ and $\left\{u_{h}, \boldsymbol{p}_{h}\right\}$ be the solutions of (5) and (6), respectively. Assume that $u, u_{t} \in H^{3}(\Omega)$ holds. Then we have

$$
\begin{aligned}
& \left\|I_{h} u-u_{h}\right\|_{1, h} \leq C h^{2}\left[\|u\|_{2}^{2}+\|\boldsymbol{p}\|_{2}^{2}+\int_{0}^{t}\left(\|u\|_{2}^{2}+\left\|u_{t}\right\|_{2}^{2}+\left\|\boldsymbol{p}_{t}\right\|_{2}^{2}\right) d s\right]^{1 / 2}, \\
& \left\|\boldsymbol{\Pi}_{h} \boldsymbol{p}-\boldsymbol{p}_{h}\right\|_{0} \leq C h^{2}\left\{\|\boldsymbol{p}\|_{2}+\left[\|u\|_{2}^{2}+\|\boldsymbol{p}\|_{2}^{2}+\int_{0}^{t}\left(\|u\|_{2}^{2}+\left\|u_{t}\right\|_{2}^{2}+\left\|\boldsymbol{p}_{t}\right\|_{2}^{2}\right) d s\right]^{1 / 2}\right\} .
\end{aligned}
$$

Proof We decompose the error as follows: $u-u_{h}=\left(u-I_{h} u\right)+\left(I_{h} u-u_{h}\right) \triangleq \eta+\xi, \boldsymbol{p}-\boldsymbol{p}_{h}=$ $\left(\boldsymbol{p}-\boldsymbol{\Pi}_{h} \boldsymbol{p}\right)+\left(\boldsymbol{\Pi}_{h} \boldsymbol{p}-\boldsymbol{p}_{h}\right) \triangleq \boldsymbol{\rho}+\boldsymbol{\theta}$. Subtracting (6) from (5) gives

$$
\left\{\begin{aligned}
\text { (a) } \quad\left(\xi_{t}, \varphi_{h}\right)-\left(\boldsymbol{\theta}, \nabla_{h} \varphi_{h}\right)_{h}= & -\left(\eta_{t}, \varphi_{h}\right)+\left(\boldsymbol{\rho}, \nabla_{h} \varphi_{h}\right)_{h}-\left(f(u)-f\left(u_{h}\right), \varphi_{h}\right) \\
& -\sum_{K} \int_{\partial K} \boldsymbol{p} \cdot \boldsymbol{n} \varphi_{h} d s, \\
\text { (b) } \quad\left(\boldsymbol{\theta}, \boldsymbol{w}_{h}\right)+\left(\nabla_{h} \xi, \boldsymbol{w}_{h}\right)_{h}= & -\left(\boldsymbol{\rho}, \boldsymbol{w}_{h}\right)-\left(\nabla_{h} \eta, \boldsymbol{w}_{h}\right)_{h} .
\end{aligned}\right.
$$

On the one hand, taking $\left\{\varphi_{h}, \boldsymbol{w}_{h}\right\}=\left\{\xi_{t}, \nabla_{h} \xi_{t}\right\}$ in the equations of (15), we have

$$
\begin{aligned}
\left\|\xi_{t}\right\|_{0}^{2}+\frac{1}{2} \frac{d}{d t}\left\|\nabla_{h} \xi\right\|_{0}^{2}= & -\left(\eta_{t}, \xi_{t}\right)-\left(\nabla_{h} \eta, \nabla_{h} \xi_{t}\right)_{h}-\left(f(u)-f\left(u_{h}\right), \xi_{t}\right) \\
& -\sum_{K} \int_{\partial K} \boldsymbol{p} \cdot \boldsymbol{n} \xi_{t} d s .
\end{aligned}
$$


Firstly, it is easy to check that

$$
\left|\left(\eta_{t}, \xi_{t}\right)\right| \leq C\left\|\eta_{t}\right\|_{0}\left\|\xi_{t}\right\|_{0} \leq C h^{4}\left\|u_{t}\right\|_{2}^{2}+\frac{1}{2}\left\|\xi_{t}\right\|_{0}^{2}
$$

and by use of (3) and (4), respectively, we derive

$$
\begin{aligned}
& \left(\nabla_{h} \eta, \nabla_{h} \xi_{t}\right)_{h}=0, \\
& \sum_{K} \int_{\partial K} \boldsymbol{p} \cdot \boldsymbol{n} \xi_{t} d s=\frac{d}{d t} \sum_{K} \int_{\partial K} \boldsymbol{p} \cdot \boldsymbol{n} \xi d s-\sum_{K} \int_{\partial K} \boldsymbol{p}_{t} \cdot \boldsymbol{n} \xi d s \\
& \leq C h^{4}\left\|\boldsymbol{p}_{t}\right\|_{2}^{2}+C\|\xi\|_{1, h}^{2}+\frac{d}{d t} \sum_{K} \int_{\partial K} \boldsymbol{p} \cdot \boldsymbol{n} \xi d s .
\end{aligned}
$$

Secondly, by virtue of (12) and (7), we have

$$
\begin{aligned}
\left(f^{\prime}(u) u_{t}-f^{\prime}\left(u_{h}\right) u_{h t}, \xi\right) & \\
= & \left(3 u^{2} u_{t}-3 u_{h}^{2} u_{t}+3 u_{h}^{2} u_{t}-3 u_{h}^{2} u_{h t}-\left(u_{t}-u_{h t}\right), \xi\right) \\
= & \left(3 u_{t}\left(u^{2}-u_{h}^{2}\right), \xi\right)+\left(3 u_{h}^{2}\left(u_{t}-u_{h t}\right), \xi\right)-\left(u_{t}-u_{h t}, \xi\right) \\
\leq & C\left\|u_{t}\right\|_{0, \infty}\left\|u-u_{h}\right\|_{0}\left\|u+u_{h}\right\|_{0,4}\|\xi\|_{0,4} \\
& +C\left\|u_{t}-u_{h t}\right\|_{0}\left\|u_{h}\right\|_{0,8}^{2}\|\xi\|_{0,4}+C\left\|u_{t}-u_{h t}\right\|_{0}\|\xi\|_{0} \\
\leq & C\left\|u-u_{h}\right\|_{0}\|\xi\|_{1, h}+C\left\|u_{t}-u_{h t}\right\|_{0}\|\xi\|_{1, h}+C\left\|u_{t}-u_{h t}\right\|_{0}\|\xi\|_{0} \\
\leq & C\left(\|\eta\|_{0}^{2}+\left\|\eta_{t}\right\|_{0}^{2}\right)+C\|\xi\|_{1, h}^{2}+\frac{1}{2}\|\xi\|_{0}^{2} \\
\leq & C h^{4}\left(\|u\|_{2}^{2}+\left\|u_{t}\right\|_{2}^{2}\right)+C\left\|_{\xi \|_{1, h}}^{2}+\frac{1}{2}\right\| \xi_{t} \|_{0}^{2},
\end{aligned}
$$

which implies that

$$
\begin{aligned}
\left(f(u)-f\left(u_{h}\right), \xi_{t}\right)= & \frac{d}{d t}\left[\left(f(u)-f\left(u_{h}\right), \xi\right)\right]-\left(f^{\prime}(u) u_{t}-f^{\prime}\left(u_{h}\right) u_{h t}, \xi\right) \\
\leq & C h^{4}\left(\|u\|_{2}^{2}+\left\|u_{t}\right\|_{2}^{2}\right)+C\|\xi\|_{1, h}^{2}+\frac{1}{2}\left\|\xi_{t}\right\|_{0}^{2} \\
& +\frac{d}{d t}\left[\left(f(u)-f\left(u_{h}\right), \xi\right)\right] .
\end{aligned}
$$

Substituting (17)-(19) and (21) into (16), then integrating from 0 to $t$, together with $\xi(\boldsymbol{x}, 0)=0$, leads to

$$
\begin{aligned}
\|\xi\|_{1, h}^{2} \leq & C h^{4} \int_{0}^{t}\left(\|u\|_{2}^{2}+\left\|u_{t}\right\|_{2}^{2}+\left\|\boldsymbol{p}_{t}\right\|_{2}^{2}\right) d s+C \int_{0}^{t}\|\xi\|_{1, h}^{2} d s \\
& -2 \sum_{K} \int_{\partial K} \boldsymbol{p} \cdot \boldsymbol{n} \xi d s-2\left(f(u)-f\left(u_{h}\right), \xi\right) .
\end{aligned}
$$

Similar to (20), we derive

$$
\begin{aligned}
\left|\left(f(u)-f\left(u_{h}\right), \xi\right)\right| & \left.=\mid\left(u-u_{h}\right)\left(u^{2}+u u_{h}+u_{h}^{2}\right), \xi\right)-\left(u-u_{h}, \xi\right) \mid \\
& \leq C\left\|u-u_{h}\right\|_{0}\left(\|u\|_{0,8}^{2}+\|u\|_{0,8}\left\|u_{h}\right\|_{0,8}+\left\|u_{h}\right\|_{0,8}^{2}\right)\|\xi\|_{0,4}
\end{aligned}
$$




$$
\begin{aligned}
& +\left\|u-u_{h}\right\|_{0}\|\xi\|_{0} \\
\leq & C\left\|u-u_{h}\right\|_{0}\|\xi\|_{1, h}+\left\|u-u_{h}\right\|_{0}\|\xi\|_{0} \\
\leq & C h^{4}\|u\|_{2}^{2}+\frac{1}{8}\|\xi\|_{1, h}^{2},
\end{aligned}
$$

and by virtue of (4), we have

$$
\sum_{K} \int_{\partial K} \boldsymbol{p} \cdot \boldsymbol{n} \xi d s \leq C h^{4}\|\boldsymbol{p}\|_{2}^{2}+\frac{1}{8}\|\xi\|_{1, h}^{2}
$$

Finally, substituting (23)-(24) into (22), then applying Gronwall's inequality yields

$$
\|\xi\|_{1, h} \leq C h^{2}\left[\|u\|_{2}^{2}+\|\boldsymbol{p}\|_{2}^{2}+\int_{0}^{t}\left(\|u\|_{2}^{2}+\left\|u_{t}\right\|_{2}^{2}+\left\|\boldsymbol{p}_{t}\right\|_{2}^{2}\right) d s\right]^{1 / 2} \text {. }
$$

On the other hand, choosing $\boldsymbol{w}_{h}=\boldsymbol{\theta}$ in the second equation in (15), employing (2)-(3) and (25), we derive

$$
\|\boldsymbol{\theta}\|_{0} \leq C h^{2}\left\{\|\boldsymbol{p}\|_{2}+\left[\|u\|_{2}^{2}+\|\boldsymbol{p}\|_{2}^{2}+\int_{0}^{t}\left(\|u\|_{2}^{2}+\left\|u_{t}\right\|_{2}^{2}+\left\|\boldsymbol{p}_{t}\right\|_{2}^{2}\right) d s\right]^{1 / 2}\right\} .
$$

The proof is completed.

Remark 3 With the help of (7) and (12), we derive the estimates for $\left|\left(f(u)-f\left(u_{h}\right), \xi\right)\right|$, which are different from those in [24].

\section{Superclose analysis for a linearized Crank-Nicolson fully-discrete scheme}

In this section, we will establish a linearized Crank-Nicolson fully-discrete scheme by MFEM and analyze superclose and superconvergence results. Let $\left\{t_{n}: t_{n}=n \tau ; 0 \leq n \leq N\right\}$ be a uniform partition of $[0, T]$ with the time step $\tau=T / N, t_{n-\frac{1}{2}}=\frac{1}{2}\left(t_{n}+t_{n-1}\right)$ and $u^{n}=$ $u\left(\boldsymbol{x}, t_{n}\right)$. For a sequence of functions $\left\{\sigma^{n}\right\}_{n=0}^{N}$, we denote

$$
\begin{array}{ll}
\bar{\partial}_{t} \sigma^{n}=\frac{\sigma^{n}-\sigma^{n-1}}{\tau}, & \bar{\sigma}^{n}=\frac{\sigma^{n}+\sigma^{n-1}}{2}, \quad n=1,2, \ldots, N, \\
\hat{\sigma}^{n}=\frac{3}{2} \sigma^{n-1}-\frac{1}{2} \sigma^{n-2}, & n=2, \ldots, N .
\end{array}
$$

With these notations, the linearized Crank-Nicolson fully-discrete approximation to (5) reads as follows: for $n \geq 2$, find $\left\{U_{h}^{n}, \boldsymbol{P}_{h}^{n}\right\} \in M_{h} \times \boldsymbol{W}_{h}$ such that

$$
\begin{cases}\left(\bar{\partial}_{t} U_{h}^{n}, \varphi_{h}\right)-\left(\overline{\boldsymbol{P}}_{h}^{n}, \nabla_{h} \varphi_{h}\right)_{h}=-\left(f\left(\widehat{U}_{h}^{n}\right), \varphi_{h}\right), & \forall \varphi_{h} \in M_{h}, \\ \left(\overline{\boldsymbol{P}}_{h}^{n}, \boldsymbol{w}_{h}\right)+\left(\nabla_{h} \bar{U}_{h}^{n}, \boldsymbol{w}_{h}\right)_{h}=0, & \forall \boldsymbol{w}_{h} \in \boldsymbol{W}_{h} .\end{cases}
$$

For $n=1,\left\{U_{h}^{1}, \boldsymbol{P}_{h}^{1}\right\}$ can be determined by

$$
\begin{cases}\left(\bar{\partial}_{t} U_{h}^{h}, \varphi_{h}\right)-\left(\overline{\boldsymbol{P}}_{h}^{1}, \nabla_{h} \varphi_{h}\right)_{h}=-\left(f\left(\frac{U_{h}^{1,0}+U_{h}^{0}}{2}\right), \varphi_{h}\right), & \forall \varphi_{h} \in M_{h}, \\ \left(\overline{\boldsymbol{P}}_{h}^{1}, \boldsymbol{w}_{h}\right)+\left(\nabla_{h} \bar{U}_{h}^{1}, \boldsymbol{w}_{h}\right)_{h}=0, & \forall \boldsymbol{w}_{h} \in \boldsymbol{W}_{h},\end{cases}
$$


together with

$$
\begin{cases}\left(\frac{U_{h}^{1,0}-U_{h}^{0}}{\tau}, \varphi_{h}\right)-\left(\frac{\boldsymbol{P}_{h}^{1,0}+\boldsymbol{P}_{h}^{0}}{2}, \nabla_{h} \varphi_{h}\right)_{h}=-\left(f\left(U_{h}^{0}\right), \varphi_{h}\right), & \forall \varphi_{h} \in M_{h}, \\ \left(\frac{\boldsymbol{P}_{h}^{1,0}+\boldsymbol{P}_{h}^{0}}{2}, \boldsymbol{w}_{h}\right)+\left(\frac{\nabla_{h} U_{h}^{1,0}+\nabla_{h} U_{h}^{0}}{2}, \boldsymbol{w}_{h}\right)_{h}=0, & \forall \boldsymbol{w}_{h} \in \boldsymbol{W}_{h},\end{cases}
$$

where $U_{h}^{0}=I_{h} u_{0}$ and $\boldsymbol{P}_{h}^{0}=\Pi_{h} \nabla u_{0}$. In what follows, the pervading strategy throughout the error analysis is splitting the error into a sum of two terms:

$$
\begin{aligned}
& u^{1}-U_{h}^{1,0}=\left(u^{1}-I_{h} u^{1}\right)+\left(I_{h} u^{1}-U_{h}^{1,0}\right) \triangleq \eta^{1}+\xi^{1,0}, \\
& u^{n}-U_{h}^{n}=\left(u^{n}-I_{h} u^{n}\right)+\left(I_{h} u^{n}-U_{h}^{n}\right) \triangleq \eta^{n}+\xi^{n}, \quad n=0,1,2, \ldots, N, \\
& \boldsymbol{p}^{n}-\boldsymbol{P}_{h}^{n}=\left(\boldsymbol{p}^{n}-\boldsymbol{\Pi}_{h} \boldsymbol{p}^{n}\right)+\left(\boldsymbol{\Pi}_{h} \boldsymbol{p}^{n}-\boldsymbol{P}_{h}^{n}\right) \triangleq \boldsymbol{\rho}^{n}+\boldsymbol{\theta}^{n}, \quad n=0,1,2, \ldots, N .
\end{aligned}
$$

Now, we are ready to present our main results in the following theorem.

Theorem 3 Suppose that equations (5) have unique solutions $\left\{u^{n}, \boldsymbol{p}^{n}\right\}$ satisfying $u, u_{t} \in$ $L^{\infty}\left(0, T ; H^{3}(\Omega)\right) \cap L^{2}\left(0, T ; H^{3}(\Omega)\right), u_{t t} \in L^{\infty}\left(0, T ; H^{2}(\Omega)\right) \cap L^{2}\left(0, T ; H^{2}(\Omega)\right), u_{t t t} \in L^{\infty}(0, T ;$ $H^{1}(\Omega)$ ), then the fully-discrete equations (27)-(29) admit unique finite element solutions $\left\{U_{h}^{n}, \boldsymbol{P}_{h}^{n}\right\}$. Further, there exist $\tau_{0}>0$ and $h_{0}>0$ such that, when $\tau \leq \tau_{0}, h \leq h_{0}$,

$$
\left\|I_{h} u^{n}-U_{h}^{n}\right\|_{1, h}+\left\|\Pi_{h} \overline{\boldsymbol{p}}^{n}-\overline{\boldsymbol{P}}_{h}^{n}\right\|_{0} \leq C_{0}\left(h^{2}+\tau^{2}\right)
$$

where $C_{0}$ is a positive constant independent of $\tau$ and $h$.

Proof First of all, we prove the existences and uniqueness of solutions of system (27)-(29). If $\left\{U_{h}^{n}, \boldsymbol{P}_{h}^{n}\right\}$ is given for $n=0,1,2, \ldots, m-1$, then the fully-discrete linear equations (27) have unique solutions $\left\{U_{h}^{m}, \boldsymbol{P}_{h}^{m}\right\}$ when, and only when, the corresponding homogeneous equations

$$
\left\{\begin{array}{l}
\frac{1}{\tau}\left(\Phi_{h}, \varphi_{h}\right)-\left(\boldsymbol{\Psi}_{h}, \nabla_{h} \varphi_{h}\right)_{h}=0 \\
\left(\boldsymbol{\Psi}_{h}, \boldsymbol{w}_{h}\right)+\left(\nabla_{h} \Phi_{h}, \boldsymbol{w}_{h}\right)_{h}=0
\end{array}\right.
$$

only have zero solutions. In fact, choosing $\left\{\varphi_{h}, \boldsymbol{w}_{h}\right\}=\left\{\Phi_{h}, \nabla_{h} \Phi_{h}\right\}$ in the above equations, we have

$$
\frac{1}{\tau}\left\|\Phi_{h}\right\|_{0}^{2}+\left\|\nabla_{h} \Phi_{h}\right\|_{0}^{2}=0
$$

we immediately get $\Phi_{h}=0$. Taking $\boldsymbol{w}_{h}=\boldsymbol{\Psi}_{h}$ in the second equation of (31), we have

$$
\left\|\Psi_{h}\right\|_{0} \leq\left\|\nabla_{h} \Phi_{h}\right\|_{0}=0
$$

which implies that $\boldsymbol{\Psi}_{h}=\mathbf{0}$. Thus, the homogeneous equation (31) has only zero solutions, which implies that the solutions of (27) exist and are unique. Similarly, we can get the existence and uniqueness of the solutions of (28) and (29). 
Next, we prove estimates (30) by using mathematical induction. For any given $n \geq 1$, $t=t_{n-\frac{1}{2}}$, any given $\left\{\varphi_{h}, \boldsymbol{w}_{h}\right\} \in M_{h} \times \boldsymbol{W}_{h}$, the weak form of (5) can be rewritten as follows:

$$
\left\{\begin{aligned}
\left(\bar{\partial}_{t} u^{n}, \varphi_{h}\right)-\left(\overline{\boldsymbol{p}}^{n}, \nabla_{h} \varphi_{h}\right)_{h}= & -\left(f\left(u^{n-\frac{1}{2}}\right), \varphi_{h}\right)+\left(R_{2}^{n}, \varphi_{h}\right)-\left(\boldsymbol{R}_{3}^{n}, \nabla_{h} \varphi_{h}\right)_{h} \\
& -\sum_{K} \int_{\partial K} \boldsymbol{p}^{n-\frac{1}{2}} \cdot \boldsymbol{n} \varphi_{h} d s \\
\left(\overline{\boldsymbol{p}}^{n}, \boldsymbol{w}_{h}\right)+\left(\nabla_{h} \bar{u}^{n}, \boldsymbol{w}_{h}\right)_{h}= & \left(\boldsymbol{R}_{3}^{n}, \boldsymbol{w}_{h}\right)+\left(\nabla_{h} R_{4}^{n}, \boldsymbol{w}_{h}\right)_{h}
\end{aligned}\right.
$$

where

$$
R_{2}^{n}=\bar{\partial}_{t} u^{n}-u_{t}^{n-\frac{1}{2}}, \quad \boldsymbol{R}_{3}^{n}=\overline{\boldsymbol{p}}^{n}-\boldsymbol{p}^{n-\frac{1}{2}}, \quad R_{4}^{n}=\bar{u}^{n}-u^{n-\frac{1}{2}}
$$

In fact, by Taylor's expansion, we have

$$
\begin{aligned}
\left\|R_{2}^{n}\right\|_{0}^{2} & =(2 \tau)^{-2}\left\|\int_{t_{n-1}}^{t_{n-\frac{1}{2}}}\left(t-t_{n-1}\right)^{2} u_{t t t}(s) d s+\int_{t_{n-\frac{1}{2}}}^{t_{n}}\left(t-t_{n}\right)^{2} u_{t t t}(s) d s\right\|_{0}^{2} \\
& \leq C \tau^{3} \int_{t_{n-1}}^{t_{n}}\left\|u_{t t t}(s)\right\|_{0}^{2} d s \leq C \tau^{4}\left\|u_{t t t}\right\|_{L^{\infty}\left(0, T ; L^{2}(\Omega)\right)}^{2},
\end{aligned}
$$

and

$$
\begin{aligned}
\left\|\nabla_{h} R_{4}^{n}\right\|_{0}^{2} & =\frac{1}{4}\left\|\int_{t_{n-1}}^{t_{n-\frac{1}{2}}^{2}}\left(t-t_{n-1}\right) \nabla_{h} u_{t t}(s) d s-\int_{t_{n-\frac{1}{2}}}^{t_{n}}\left(t-t_{n}\right) \nabla_{h} u_{t t}(s) d s\right\|_{0}^{2} \\
& \leq C \tau^{3} \int_{t_{n-1}}^{t_{n}}\left\|\nabla_{h} u_{t t}(s)\right\|_{0}^{2} d s .
\end{aligned}
$$

By $U_{h}^{0}=I_{h} u_{0}$, we can derive that

$$
\left\|u_{0}-U_{h}^{0}\right\|_{0, p}=\left\|u_{0}-I_{h} u_{0}\right\|_{0, p} \leq C h^{2}\left\|u_{0}\right\|_{2, p}, \quad 1 \leq p<\infty
$$

When $n=1$, on the one hand, subtracting (29) from (32), taking $\left\{\varphi_{h}, \boldsymbol{w}_{h}\right\}=\left\{\frac{\xi^{1,0}}{\tau}, \nabla_{h} \frac{\xi^{1,0}}{\tau}\right\}$, and noting that $\xi^{0}=0$, we have

$$
\begin{aligned}
2 \tau\left\|\bar{\partial}_{t} \xi^{1,0}\right\|_{0}^{2}+\left\|\xi^{1,0}\right\|_{1, h}^{2}= & 2 \tau\left(R_{2}^{1}, \bar{\partial}_{t} \xi^{1,0}\right)-2 \tau\left(\bar{\partial}_{t} \eta^{1}, \bar{\partial}_{t} \xi^{1,0}\right) \\
& -2 \tau \sum_{K} \int_{\partial K} \boldsymbol{p}^{\frac{1}{2}} \cdot \boldsymbol{n} \bar{\partial}_{t} \xi^{1,0} d s-2 \tau\left(f\left(u^{\frac{1}{2}}\right)-f\left(U_{h}^{0}\right), \bar{\partial}_{t} \xi^{1,0}\right) \\
& -2 \tau\left(\nabla_{h} \bar{\eta}^{1}, \bar{\partial}_{t} \nabla_{h} \xi^{1,0}\right)_{h}+2 \tau\left(\nabla_{h} R_{4}^{1}, \bar{\partial}_{t} \nabla_{h} \xi^{1,0}\right)_{h} \triangleq \sum_{i=1}^{6} F_{i}
\end{aligned}
$$

By (33) and (34), $F_{1}, F_{2}, F_{6}$ can be estimated as

$$
\begin{aligned}
F_{1}+F_{2}+F_{6} \leq & C \tau\left(\left\|R_{2}^{1}\right\|_{0}^{2}+\left\|\bar{\partial}_{t} \eta^{1}\right\|_{0}^{2}\right)+\tau\left\|\bar{\partial}_{t} \xi^{1,0}\right\|_{0}^{2}+C\left\|\nabla_{h} R_{4}^{1}\right\|_{0}^{2}\left\|\nabla_{h} \xi^{1,0}\right\|_{0} \\
\leq & C \tau^{4}\left(\left\|u_{t t t}\right\|_{L^{\infty}\left(0, T ; L^{2}(\Omega)\right)}^{2}+\left\|u_{t t}\right\|_{L^{2}\left(0, T ; H^{1}(\Omega)\right)}^{2}\right) \\
& +C h^{4}\left\|u_{t}\right\|_{L^{2}\left(0, T ; H^{2}(\Omega)\right)}^{2}+\tau\left\|\bar{\partial}_{t} \xi^{1,0}\right\|_{0}^{2}+\frac{1}{4}\left\|\xi^{1,0}\right\|_{1, h}^{2} .
\end{aligned}
$$


And by use of (3) and (4), respectively, we can see that

$$
F_{5}=0, \quad F_{3}=2 \sum_{K} \int_{\partial K} \boldsymbol{p}^{\frac{1}{2}} \cdot \boldsymbol{n} \xi^{1,0} d s \leq C h^{4}\|\boldsymbol{p}\|_{L^{\infty}\left(0, T ; H^{2}(\Omega)\right)}^{2}+\frac{1}{4}\left\|\xi^{1,0}\right\|_{1, h}^{2} .
$$

To estimate $F_{4}$ and circumvent the time step size restrictions caused by the nonlinear term $f(u)$, we introduce a novel splitting technique which splits $F_{4}$ into different parts, and by use of (35), we derive

$$
\begin{aligned}
&\left|\left(f\left(u_{0}\right)-f\left(U_{h}^{0}\right), \bar{\partial}_{t} \xi^{1,0}\right)\right| \\
&=\left|\left(\left(\left(u_{0}\right)^{3}-u_{0}\right)-\left(\left(U_{h}^{0}\right)^{3}-U_{h}^{0}\right), \bar{\partial}_{t} \xi^{1,0}\right)\right| \\
&=\mid\left(\left(u_{0}-U_{h}^{0}\right)^{3}, \bar{\partial}_{t} \xi^{1,0}\right)-3\left(\left(u_{0}-U_{h}^{0}\right)^{2} u_{0}, \bar{\partial}_{t} \xi^{1,0}\right) \\
& \quad+3\left(\left(u_{0}-U_{h}^{0}\right) u_{0}^{2}, \bar{\partial}_{t} \xi^{1,0}\right)-\left(u^{0}-U_{h}^{0}, \bar{\partial}_{t} \xi^{1,0}\right) \mid \\
& \leq C\left\|u_{0}-U_{h}^{0}\right\|_{0,6}^{3}\left\|\bar{\partial}_{t} \xi^{1,0}\right\|_{0}+C\left\|u_{0}-U_{h}^{0}\right\|_{0,4}^{2}\left\|u_{0}\right\|_{0, \infty}\left\|\bar{\partial}_{t} \xi^{1,0}\right\|_{0} \\
& \quad+C\left\|u_{0}-U_{h}^{0}\right\|_{0}\left\|u_{0}\right\|_{0, \infty}^{2}\left\|\bar{\partial}_{t} \xi^{1,0}\right\|_{0}+C h^{2}\left\|u_{0}\right\|_{2}\left\|\bar{\partial}_{t} \xi^{1,0}\right\|_{0} \\
& \leq C h^{4}+\frac{1}{2}\left\|\bar{\partial}_{t} \xi^{1,0}\right\|_{0}^{2} .
\end{aligned}
$$

Thus,

$$
\begin{aligned}
F_{4} & =-2 \tau\left(f\left(u^{\frac{1}{2}}\right)-f\left(u_{0}\right), \bar{\partial}_{t} \xi^{1,0}\right)-2 \tau\left(f\left(u_{0}\right)-f\left(U_{h}^{0}\right), \bar{\partial}_{t} \xi^{1,0}\right) \\
& \leq C \tau\left\|f\left(u^{\frac{1}{2}}\right)-f\left(u_{0}\right)\right\|_{0}^{2}+\frac{\tau}{2}\left\|\bar{\partial}_{t} \xi^{1,0}\right\|_{0}^{2}-2 \tau\left(f\left(u_{0}\right)-f\left(U_{h}^{0}\right), \bar{\partial}_{t} \xi^{1,0}\right) \\
& \leq C \tau^{3}\left\|u_{t}\right\|_{L^{\infty}\left(0, T ; L^{2}(\Omega)\right)}^{2}+C h^{4}+\tau\left\|\bar{\partial}_{t} \xi^{1,0}\right\|_{0}^{2} .
\end{aligned}
$$

Substituting the above estimates $F_{i}(i=1,2, \ldots, 6)$ into (36), there exist $C_{1}, \tau_{1}$ such that when $\tau \leq \tau_{1}$, there holds

$$
\left\|\xi^{1,0}\right\|_{1, h}^{2} \leq C_{1}\left(h^{4}+\tau^{3}\right)
$$

and with the help of (12), we have

$$
\left\|\xi^{1,0}\right\|_{0,2 k}^{2} \leq 1
$$

when $\tau \leq \tau_{2}=\sqrt[3]{1 / 2 C C_{1}}, h \leq h_{1}=\sqrt[4]{1 / 2 C C_{1}}$.

On the other hand, we estimate $\left\|\xi^{1}\right\|_{1, h}^{2}$. Subtracting (28) from (32), taking $\left\{\varphi_{h}, \boldsymbol{w}_{h}\right\}=$ $\left\{\frac{\xi^{1}}{\tau}, \nabla_{h} \frac{\xi^{1}}{\tau}\right\}$, and noting that $\xi^{0}=0$, we have

$$
\begin{aligned}
2 \tau\left\|\bar{\partial}_{t} \xi^{1}\right\|_{0}^{2}+\left\|\xi^{1}\right\|_{1, h}^{2}= & 2 \tau\left(R_{2}^{1}, \bar{\partial}_{t} \xi^{1}\right)-2 \tau\left(\bar{\partial}_{t} \eta^{1}, \bar{\partial}_{t} \xi^{1}\right) \\
& -2 \tau \sum_{K} \int_{\partial K} \boldsymbol{p}^{\frac{1}{2}} \cdot \boldsymbol{n} \bar{\partial}_{t} \xi^{1} d s-2 \tau\left(f\left(u^{\frac{1}{2}}\right)-f\left(\frac{U_{h}^{1,0}+U_{h}^{0}}{2}\right), \bar{\partial}_{t} \xi^{1}\right) \\
& -2 \tau\left(\nabla_{h} \bar{\eta}^{1}, \bar{\partial}_{t} \nabla_{h} \xi^{1}\right)_{h}+2 \tau\left(\nabla_{h} R_{4}^{1}, \bar{\partial}_{t} \nabla_{h} \xi^{1}\right)_{h} \triangleq \sum_{i=1}^{6} G_{i}
\end{aligned}
$$


Similar to the estimates $F_{i}(i=1,2,3,5,6), G_{i}(i=1,2,3,5,6)$ can be estimated as follows:

$$
\begin{gathered}
G_{1}+G_{2}+G_{6} \leq C \tau^{4}\left(\left\|u_{t t t}\right\|_{L^{\infty}\left(0, T ; L^{2}(\Omega)\right)}^{2}+\left\|\boldsymbol{p}_{t t}\right\|_{L^{\infty}\left(0, T ; L^{2}(\Omega)\right)}^{2}\right) \\
+C h^{4}\left\|u_{t}\right\|_{L^{2}\left(0, T ; H^{2}(\Omega)\right)}^{2}+\tau\left\|\bar{\partial}_{t} \xi^{1}\right\|_{0}^{2}+\frac{1}{4}\left\|\xi^{1}\right\|_{1, h^{\prime}}^{2}, \\
G_{3} \leq C h^{4}\|\boldsymbol{p}\|_{L^{\infty}\left(0, T ; H^{2}(\Omega)\right)}^{2}+\frac{1}{4}\left\|\xi^{1}\right\|_{1, h^{\prime}}^{2} \quad G_{5}=0 .
\end{gathered}
$$

Now we estimate $G_{4}$. In order to get rid of the time step restriction, we rewrite $G_{4}$ as follows:

$$
\begin{aligned}
G_{4}= & -2 \tau\left(f\left(u^{\frac{1}{2}}\right)-f\left(\frac{u^{1}+u^{0}}{2}\right), \bar{\partial}_{t} \xi^{1}\right) \\
& -2 \tau\left(f\left(\frac{u^{1}+u^{0}}{2}\right)-f\left(\frac{U_{h}^{1,0}+U_{h}^{0}}{2}\right), \bar{\partial}_{t} \xi^{1}\right) \\
= & -2 \tau\left(f\left(u^{\frac{1}{2}}\right)-f\left(\frac{u^{1}+u^{0}}{2}\right), \bar{\partial}_{t} \xi^{1}\right) \\
& -2 \tau\left(\left(\frac{u^{1}+u^{0}}{2}-\frac{U_{h}^{1,0}+U_{h}^{0}}{2}\right)^{3}, \bar{\partial}_{t} \xi^{1}\right) \\
& +6 \tau\left(\left(\frac{u^{1}+u^{0}}{2}-\frac{U_{h}^{1,0}+U_{h}^{0}}{2}\right)^{2} \frac{u^{1}+u^{0}}{2}, \bar{\partial}_{t} \xi^{1}\right) \\
& -6 \tau\left(\left(\frac{u^{1}+u^{0}}{2}-\frac{U_{h}^{1,0}+U_{h}^{0}}{2}\right)\left(\frac{u^{1}+u^{0}}{2}\right)^{2}, \bar{\partial}_{t} \xi^{1}\right) \\
& +2 \tau\left(\frac{u^{1}+u^{0}}{2}-\frac{U_{h}^{1,0}+U_{h}^{0}}{2}, \bar{\partial}_{t} \xi^{1}\right) \triangleq \sum_{i=1}^{5} G_{4 i}, \\
G_{41} \leq & C \tau\left\|f\left(u^{\frac{1}{2}}\right)-f\left(\frac{u^{1}+u^{0}}{2}\right)\right\|_{0}^{2}+\frac{\tau}{2}\left\|\bar{\partial}_{t} \xi^{1}\right\|_{0}^{2} \leq C \tau^{4}+\frac{\tau}{2}\left\|\bar{\partial}_{t} \xi^{1}\right\|_{0}^{2} .
\end{aligned}
$$

By use of (37) and (38), and noting that $\xi^{0}=0$, we derive

$$
\begin{aligned}
\sum_{i=2}^{5} G_{4 i} \leq & C \tau\left(\left\|\eta^{1}\right\|_{0,6}^{3}+\left\|\xi^{1,0}\right\|_{0,6}^{3}+\left\|\eta^{0}\right\|_{0,6}^{3}\right)\left\|\bar{\partial}_{t} \xi^{1}\right\|_{0} \\
& +C \tau\left(\left\|\eta^{1}\right\|_{0,4}^{2}+\left\|\xi^{1,0}\right\|_{0,4}^{2}+\left\|\eta^{0}\right\|_{0,4}^{2}\right)\left\|\frac{u^{1}+u^{0}}{2}\right\|_{0, \infty}\left\|\bar{\partial}_{t} \xi^{1}\right\|_{0} \\
& +C \tau\left(\left\|\eta^{1}\right\|_{0}+\left\|\xi^{1,0}\right\|_{0}+\left\|\eta^{0}\right\|_{0}\right)\left\|\frac{u^{1}+u^{0}}{2}\right\|_{0, \infty}^{2}\left\|\bar{\partial}_{t} \xi^{1}\right\|_{0} \\
& +C \tau\left(\left\|\eta^{1}\right\|_{0}+\left\|\xi^{1,0}\right\|_{0}+\left\|\eta^{0}\right\|_{0}\right)\left\|\bar{\partial}_{t} \xi^{1}\right\|_{0} \\
\leq & C\left(\tau^{4}+h^{4}\right)+\frac{\tau}{2}\left\|\bar{\partial}_{t} \xi^{1}\right\|_{0}^{2},
\end{aligned}
$$

which together with (40) implies that

$$
G_{4} \leq C\left(\tau^{4}+h^{4}\right)+\tau\left\|\bar{\partial}_{t} \xi^{1}\right\|_{0}^{2}
$$


Substituting the above estimates $G_{i}(i=1,2, \ldots, 6)$ into (39), then by Gronwall's inequality, there exist $C_{2}, \tau_{3}>0$, when $\tau \leq \tau_{3}$, such that

$$
\left\|\xi^{1}\right\|_{1, h}^{2} \leq C_{2}\left(h^{4}+\tau^{4}\right)
$$

Moreover, subtracting the second equation of (28) from the second equation of (32) and choosing $\boldsymbol{w}_{h}=\overline{\boldsymbol{\theta}}^{1}$, we have

$$
\left\|\overline{\boldsymbol{\theta}}^{1}\right\|_{0}^{2}=-\left(\nabla_{h} \xi^{1}, \overline{\boldsymbol{\theta}}^{1}\right)_{h}-\left(\overline{\boldsymbol{\rho}}^{1}, \overline{\boldsymbol{\theta}}^{1}\right)-\left(\nabla_{h} \eta^{1}, \overline{\boldsymbol{\theta}}^{1}\right)_{h}+\left(\boldsymbol{R}_{3}^{1}, \overline{\boldsymbol{\theta}}^{1}\right)+\left(\nabla_{h} \boldsymbol{R}_{4}^{1}, \overline{\boldsymbol{\theta}}^{1}\right)_{h} .
$$

Using the Cauchy-Schwarz inequality, there holds

$$
\begin{aligned}
& \left|\left(\nabla_{h} \xi^{1}, \overline{\boldsymbol{\theta}}^{1}\right)_{h}+\left(\boldsymbol{R}_{3}^{1}, \overline{\boldsymbol{\theta}}^{1}\right)+\left(\nabla_{h} R_{4}^{1}, \overline{\boldsymbol{\theta}}^{1}\right)_{h}\right| \\
& \quad \leq\left(\left\|\nabla_{h} \xi^{1}\right\|_{0}+\left\|\boldsymbol{R}_{3}^{1}\right\|_{0}+\left\|\nabla_{h} R_{4}^{1}\right\|_{0}\right)\left\|\overline{\boldsymbol{\theta}}^{1}\right\|_{0} .
\end{aligned}
$$

Employing (2) and (3), respectively, we can see that

$$
\left|\left(\overline{\boldsymbol{\rho}}^{1}, \overline{\boldsymbol{\theta}}^{1}\right)\right| \leq C h^{2}\left\|\overline{\boldsymbol{p}}^{1}\right\|_{2}\left\|\overline{\boldsymbol{\theta}}^{1}\right\|_{0}
$$

and

$$
\left|\left(\nabla_{h} \eta^{1}, \overline{\boldsymbol{\theta}}^{1}\right)_{h}\right|=0
$$

respectively. With the above estimates of (33)-(34) and (41)-(45), there exist $C_{3}, \tau_{4}>0$, when $\tau \leq \tau_{4}$, such that

$$
\left\|\overline{\boldsymbol{\theta}}^{1}\right\|_{0}^{2} \leq C_{3}\left(h^{4}+\tau^{4}\right)
$$

Now, we assume that (30) holds for $m \leq n-1$. By use of (12) again, we have

$$
\left\|\xi^{m}\right\|_{0,2 k}^{2} \leq C C_{0}\left(h^{4}+\tau^{4}\right)
$$

which implies that

$$
\left\|\xi^{m}\right\|_{0,2 k}^{2} \leq 1
$$

when $\tau \leq \tau_{5}=\sqrt[4]{1 / 2 C C_{0}}, h \leq h_{2}=\sqrt[4]{1 / 2 C C_{0}}$.

In the following, we prove that (30) also holds for $m=n$. From (27) and (32), we have

$$
\left\{\begin{aligned}
\text { (a) } \quad\left(\bar{\partial}_{t} \xi^{n}, \varphi_{h}\right)-\left(\overline{\boldsymbol{\theta}}^{n}, \nabla_{h} \varphi_{h}\right)_{h}= & \left(R_{2}^{n}, \varphi_{h}\right)-\left(\boldsymbol{R}_{3}^{n}, \nabla_{h} \varphi_{h}\right)_{h}-\left(\bar{\partial}_{t} \eta^{n}, \varphi_{h}\right) \\
& +\left(\overline{\boldsymbol{\rho}}^{n}, \nabla_{h} \varphi_{h}\right)_{h}-\sum_{K} \int_{\partial K} \boldsymbol{p}^{n-\frac{1}{2}} \cdot \boldsymbol{n} \varphi_{h} d s \\
& -\left(f\left(u^{n-\frac{1}{2}}\right)-f\left(\widehat{U}_{h}^{n}\right), \varphi_{h}\right), \\
\text { (b) } \quad\left(\overline{\boldsymbol{\theta}}^{n}, \boldsymbol{w}_{h}\right)+\left(\nabla_{h} \xi^{n}, \boldsymbol{w}_{h}\right)_{h}= & -\left(\overline{\boldsymbol{\rho}}^{n}, \boldsymbol{w}_{h}\right)-\left(\nabla_{h} \eta^{n}, \boldsymbol{w}_{h}\right)_{h} \\
& +\left(\boldsymbol{R}_{3}^{n}, \boldsymbol{w}_{h}\right)+\left(\nabla_{h} R_{4}^{n}, \boldsymbol{w}_{h}\right)_{h} .
\end{aligned}\right.
$$


Choosing $\left\{\varphi_{h}, \boldsymbol{w}_{h}\right\}=\left\{\bar{\partial}_{t} \xi^{n}, \bar{\partial}_{t} \nabla_{h} \xi^{n}\right\}$ in (49), replacing $n$ by $i$, and summing up from 2 to $n$, we can derive the following error equation:

$$
\begin{aligned}
2 \tau \sum_{i=2}^{n}\left\|\bar{\partial}_{t} \xi^{i}\right\|_{0}^{2}+\left\|\xi^{n}\right\|_{1, h}^{2} \\
=\left\|\xi^{1}\right\|_{1, h}^{2}+2 \tau \sum_{i=2}^{n}\left(R_{2}^{i}, \bar{\partial}_{t} \xi^{i}\right)-2 \tau \sum_{i=2}^{n}\left(\bar{\partial}_{t} \eta^{i}, \bar{\partial}_{t} \xi^{i}\right) \\
\quad-2 \tau \sum_{i=2}^{n} \sum_{K} \int_{\partial K} \boldsymbol{p}^{i-\frac{1}{2}} \cdot \boldsymbol{n} \bar{\partial}_{t} \xi^{i} d s-2 \tau \sum_{i=2}^{n}\left(f\left(u^{i-\frac{1}{2}}\right)-f\left(\widehat{U}_{h}^{i}\right), \bar{\partial}_{t} \xi^{i}\right) \\
\quad-2 \tau \sum_{i=2}^{n}\left(\nabla_{h} \bar{\eta}^{i}, \bar{\partial}_{t} \nabla_{h} \xi^{i}\right)_{h}+2 \tau \sum_{i=2}^{n}\left(\nabla_{h} R_{4}^{i}, \bar{\partial}_{t} \nabla_{h} \xi^{i}\right)_{h} \triangleq \sum_{j=1}^{7} E_{j} .
\end{aligned}
$$

Now we estimate the terms on the right-hand side of (50).

Similar to (33)-(34), we obtain

$$
\left\|\bar{\partial}_{t} \nabla_{h} R_{4}^{i}\right\|_{0}^{2} \leq C \tau^{4}\left\|u_{t t t}\right\|_{L^{\infty}\left(0, T ; H^{1}(\Omega)\right)}^{2}
$$

and

$$
\left(\nabla_{h} R_{4}^{i}, \bar{\partial}_{t} \nabla_{h} \xi^{i}\right)_{h}=\bar{\partial}_{t}\left(\nabla_{h} R_{4}^{i}, \nabla_{h} \xi^{i}\right)_{h}-\left(\bar{\partial}_{t} \nabla_{h} R_{4}^{i}, \nabla_{h} \xi^{i-1}\right)_{h}
$$

then $E_{2}, E_{3}$, and $E_{7}$ can be estimated as

$$
\begin{aligned}
E_{2}+E_{3} & \leq C \tau \sum_{i=2}^{n}\left(\left\|R_{2}^{i}\right\|_{0}^{2}+\left\|\bar{\partial}_{t} \eta^{i}\right\|_{0}^{2}\right)+\tau \sum_{i=2}^{n}\left\|\bar{\partial}_{t} \xi^{i}\right\|_{0}^{2} \\
& \leq C \tau^{4}\left\|u_{t t t}\right\|_{L^{\infty}\left(0, T ; L^{2}(\Omega)\right)}^{2}+C h^{4}\left\|u_{t}\right\|_{L^{2}\left(0, T ; L^{2}(\Omega)\right)}^{2}+\tau \sum_{i=2}^{n}\left\|\bar{\partial}_{t} \xi^{i}\right\|_{0}^{2}
\end{aligned}
$$

and

$$
E_{7} \leq C \tau^{4}\left(\left\|u_{t t}\right\|_{L^{\infty}\left(0, T ; H^{1}(\Omega)\right)}^{2}+\left\|u_{t t t}\right\|_{L^{\infty}\left(0, T ; H^{1}(\Omega)\right)}^{2}\right)+C\left\|\xi^{1}\right\|_{1, h}^{2}+C \tau \sum_{i=2}^{n}\left\|\xi^{i-1}\right\|_{1, h^{\prime}}^{2}
$$

respectively. Note that

$$
\begin{aligned}
\sum_{K} \int_{\partial K} \boldsymbol{p}^{i-\frac{1}{2}} \cdot \boldsymbol{n} \bar{\partial}_{t} \xi^{i} d s= & \frac{1}{\tau}\left(\sum_{K} \int_{\partial K} \boldsymbol{p}^{i-\frac{1}{2}} \cdot \boldsymbol{n} \xi^{i} d s-\sum_{K} \int_{\partial K} \boldsymbol{p}^{i-\frac{3}{2}} \cdot \boldsymbol{n} \xi^{i-1} d s\right) \\
& -\sum_{K} \int_{\partial K} \frac{\boldsymbol{p}^{n-\frac{1}{2}}-\boldsymbol{p}^{n-\frac{3}{2}}}{\tau} \cdot \boldsymbol{n} \xi^{n-1} d s \\
\leq & \frac{1}{\tau}\left(\sum_{K} \int_{\partial K} \boldsymbol{p}^{i-\frac{1}{2}} \cdot \boldsymbol{n} \xi^{i} d s-\sum_{K} \int_{\partial K} \boldsymbol{p}^{i-\frac{3}{2}} \cdot \boldsymbol{n} \xi^{i-1} d s\right) \\
& +C h^{4}\left\|\boldsymbol{p}_{t}\right\|_{L^{2}\left(0, T ; H^{2}(\Omega)\right)}^{2}+C\left\|\xi^{n-1}\right\|_{1, h^{\prime}}^{2}
\end{aligned}
$$


which together with (4) yields

$$
\begin{aligned}
E_{4} \leq & -\left(\sum_{K} \int_{\partial K} \boldsymbol{p}^{n-\frac{1}{2}} \cdot \boldsymbol{n} \xi^{n} d s-\sum_{K} \int_{\partial K} \boldsymbol{p}^{\frac{1}{2}} \cdot \boldsymbol{n} \xi^{1} d s\right) \\
& +C h^{4}\left\|\boldsymbol{p}_{t}\right\|_{L^{2}\left(0, T ; H^{2}(\Omega)\right)}^{2}+C \sum_{i=2}^{n}\left\|\xi^{i-1}\right\|_{1, h}^{2} \\
\leq & C h^{4}\left\|\boldsymbol{p}_{t}\right\|_{L^{2}\left(0, T ; H^{2}(\Omega)\right)}^{2}+C \tau \sum_{i=2}^{n}\left\|\xi^{n-1}\right\|_{1, h}^{2}+\frac{2}{5}\left\|\xi^{n}\right\|_{1, h}^{2}+C\left\|\xi^{1}\right\|_{1, h}^{2} .
\end{aligned}
$$

By use of (47)-(48), with the same argument of $G_{4}$, we can see that

$$
E_{5} \leq C \tau^{4}+C h^{4}+\tau \sum_{i=2}^{n}\left\|\bar{\partial}_{t} \xi^{i}\right\|_{0}^{2} .
$$

Thanks to (3), we have

$$
E_{6}=0 .
$$

Then, substituting the above estimates $E_{j}(j=1,2, \ldots, 7)$ into (50) and employing the discrete Gronwall's inequality, there exist $C_{4}, \tau_{6}>0$, when $\tau \leq \tau_{6}$, such that

$$
\left\|\xi^{n}\right\|_{1, h}^{2} \leq C_{4}\left(h^{4}+\tau^{4}\right)
$$

Let $\boldsymbol{w}_{h}=\overline{\boldsymbol{\theta}}^{n}$ in the second equation of (49), and with similar arguments of (42)-(46), we deduce that

$$
\left\|\overline{\boldsymbol{\theta}}^{n}\right\|_{0}^{2} \leq C_{5}\left(h^{4}+\tau^{4}\right)
$$

where $C_{5}$ is independent of $h$ and $\tau$. Thus, (30) holds for $m=n$ if we take $C_{0} \geq \sum_{i=1}^{5} C_{i}$, $i=1,2, \ldots, 5, h_{0} \leq \min _{1 \leq i \leq 2} h_{i}$, and $\tau_{0} \leq \min _{1 \leq i \leq 6} \tau_{i}$. The induction is closed and the proof of Theorem 3 is completed.

\section{Superconvergence analysis}

In order to get global superconvergence results, we merge four small elements into a big one $\bar{K}=\bigcup_{i=1}^{4} K_{i}$ and define the following interpolated postprocessing operators $I_{2 h}$ and $\Pi_{2 h}$ as in [39]. The operators $I_{2 h}$ and $\Pi_{2 h}$ have the following properties, respectively:

$$
\begin{cases}I_{2 h} I_{h} u=I_{2 h} u, & \forall u \in H^{2}(\Omega), \\ \left\|I_{2 h} u-u\right\|_{1, h} \leq C h^{2}|u|_{3}, & \forall u \in H^{3}(\Omega), \\ \left\|I_{2 h} u_{h}\right\|_{1, h} \leq C\left\|u_{h}\right\|_{1, h}, & \forall u_{h} \in M_{h},\end{cases}
$$

and

$$
\begin{cases}\boldsymbol{\Pi}_{2 h} \boldsymbol{\Pi}_{h} \boldsymbol{p}=\boldsymbol{\Pi}_{2 h} \boldsymbol{p}, & \forall \boldsymbol{p} \in\left(H^{1}(\Omega)\right)^{2}, \\ \left\|\boldsymbol{\Pi}_{2 h} \boldsymbol{p}-\boldsymbol{p}\right\|_{0} \leq C h^{2}\|\boldsymbol{p}\|_{2}, & \forall \boldsymbol{p} \in\left(H^{2}(\Omega)\right)^{2}, \\ \left\|\boldsymbol{\Pi}_{2 h} \boldsymbol{p}_{h}\right\|_{0} \leq C\left\|\boldsymbol{p}_{h}\right\|_{0}, & \forall \boldsymbol{p}_{h} \in \boldsymbol{W}_{h} .\end{cases}
$$

Then we can deduce the following global superconvergence results. 
Theorem 4 Under the assumptions of Theorems 2 and 3, respectively, we have the following results:

(a) For a semi-discrete case,

$$
\begin{aligned}
& \left\|I_{2 h} u_{h}-u\right\|_{1, h} \\
& \quad \leq C h^{2}\left\{\|u\|_{3}+\left[\|u\|_{2}^{2}+\|\boldsymbol{p}\|_{2}^{2}+\int_{0}^{t}\left(\|u\|_{2}^{2}+\left\|u_{t}\right\|_{2}^{2}+\left\|\boldsymbol{p}_{t}\right\|_{2}^{2}\right) d s\right]^{1 / 2}\right\}, \\
& \left\|\boldsymbol{\Pi}_{2 h} \boldsymbol{p}_{h}-\boldsymbol{p}\right\|_{0} \\
& \quad \leq C h^{2}\left\{\|\boldsymbol{p}\|_{2}+\left[\|u\|_{2}^{2}+\|\boldsymbol{p}\|_{2}^{2}+\int_{0}^{t}\left(\|u\|_{2}^{2}+\left\|u_{t}\right\|_{2}^{2}+\left\|\boldsymbol{p}_{t}\right\|_{2}^{2}\right) d s\right]^{1 / 2}\right\} .
\end{aligned}
$$

(b) For a Crank-Nicolson fully-discrete case,

$$
\left\|I_{2 h} U_{h}^{n}-u^{n}\right\|_{1, h}+\left\|\boldsymbol{\Pi}_{2 h} \overline{\boldsymbol{P}}_{h}^{n}-\overline{\boldsymbol{p}}^{n}\right\|_{0}=O\left(h^{2}+\tau^{2}\right) .
$$

Proof In fact, by the triangle inequality and (53), we have

$$
\begin{aligned}
\left\|I_{2 h} u_{h}-u\right\|_{1, h} & \leq\left\|I_{2 h} u_{h}-I_{2 h} I_{h} u\right\|_{1, h}+\left\|I_{2 h} I_{h} u-u\right\|_{1, h} \\
& =\left\|I_{2 h}\left(u_{h}-I_{h} u\right)\right\|_{1, h}+\left\|I_{2 h} u-u\right\|_{1, h} \\
& \leq C\left\|u_{h}-I_{h} u\right\|_{1, h}+C h^{2}\|u\|_{3} \\
& \leq C h^{2}\left\{\|u\|_{3}+\left[\|u\|_{2}^{2}+\|\boldsymbol{p}\|_{2}^{2}+\int_{0}^{t}\left(\|u\|_{2}^{2}+\left\|u_{t}\right\|_{2}^{2}+\left\|\boldsymbol{p}_{t}\right\|_{2}^{2}\right) d s\right]^{1 / 2}\right\},
\end{aligned}
$$

and with the similar arguments, we also derive the results (56) and (57). This completes the proof.

Remark 4 For the linearized Crank-Nicolson scheme, we derive the required superclose and superconvergence results unconditionally due to two key methods: one is a new splitting technique for the consistency error, such as $E_{4}$. If we use traditional technique in $E_{4}$, when $\tau=O(h)$, by (4) and the inverse inequality, we have

$$
\begin{aligned}
E_{4} & =\sum_{K} \int_{\partial K} \boldsymbol{p}^{n-\frac{1}{2}} \cdot \boldsymbol{n} \bar{\partial}_{t} \xi^{n} d s \\
& \leq C h^{2}\left\|\boldsymbol{p}^{n-\frac{1}{2}}\right\|_{2}\left\|\bar{\partial}_{t} \xi^{n}\right\|_{1, h} \\
& \leq C h\left\|\boldsymbol{p}^{n-\frac{1}{2}}\right\|_{2}\left\|\xi^{n}-\xi^{n-1}\right\|_{1, h}
\end{aligned}
$$

only the estimate of order $O(h)$ is derived; another is the sharp estimates for nonlinear terms by using (12), which leads to weakened restriction for the reaction term $f(u)$ to be more "nonlinear". From these points of view, it is not an easy thing to develop a good scheme and get superclose estimates. 


\section{Numerical results}

In this section, we provide a numerical example to verify the correctness of the theoretical analysis. Consider the following parabolic equation:

$$
\begin{cases}u_{t}-\Delta u-f(u)=g(\boldsymbol{x}, t), & (\boldsymbol{x}, t) \in \Omega \times(0, T] \\ u=0, & (\boldsymbol{x}, t) \in \partial \Omega \times(0, T], \\ u(\boldsymbol{x}, 0)=u_{0}, & \boldsymbol{x} \in \Omega,\end{cases}
$$

with $\Omega=[0,1] \times[0,1], f(u)=u^{3}-u$, and $g(\boldsymbol{x}, t)$ is chosen corresponding to the exact solution $u=e^{t} x y(1-x)(1-y)$.

We solve the above equation by the linearized Crank-Nicolson MFEM (27)-(29) and divide $\Omega$ into $m \times m$ uniform rectangles with $m \times m=20 \times 20,40 \times 40,80 \times 80$. The error results with respect to $t=0.25,0.50,0.75$, and 1 are listed in Tables $1-8$. Tables $1-4$ show that when $h \rightarrow 0,\left\|u^{n}-U^{n}\right\|_{1, h}$ is convergent to the optimal order $O(h)$ and $\left\|U^{n}-I_{h} u^{n}\right\|_{1, h}$ is convergent to order $O\left(h^{2}\right)$. At the same time, Tables 5-8 show that when $h \rightarrow 0,\left\|\boldsymbol{p}^{n}-\boldsymbol{P}_{h}^{n}\right\|_{0}$ is convergent to the optimal order $O(h)$ and $\left\|\boldsymbol{P}_{h}^{n}-\boldsymbol{\Pi}_{h} \boldsymbol{p}^{n}\right\|_{0}$ is convergent to the order $O\left(h^{2}\right)$. Numerical results coincide with our theoretical analysis.

Table 1 Numerical results at $t=0.25$ with $\tau=5 h$

\begin{tabular}{lllll}
\hline$m \times m$ & $\left\|u^{n}-U_{h}^{n}\right\|_{1, h}$ & Order & $\left\|U_{h}^{n}-\ln _{h} u^{n}\right\|_{1, h}$ & Order \\
\hline $20 \times 20$ & 0.0087 & - & $0.4060 \times 10^{-3}$ & - \\
$40 \times 40$ & 0.0044 & 0.9999 & $0.1012 \times 10^{-3}$ & 2.0043 \\
$80 \times 80$ & 0.0022 & 1.0000 & $0.0253 \times 10^{-3}$ & 1.9996 \\
\hline
\end{tabular}

Table 2 Numerical results at $t=0.5$ with $\tau=5 h$

\begin{tabular}{lllll}
\hline$m \times m$ & $\left\|u^{n}-U_{h}^{n}\right\|_{1, h}$ & Order & $\left\|U_{h}^{n}-\ln _{h} u^{n}\right\|_{1, h}$ & Order \\
\hline $20 \times 20$ & 0.0112 & - & $0.5226 \times 10^{-3}$ & - \\
$40 \times 40$ & 0.0056 & 0.9999 & $0.1308 \times 10^{-3}$ & 1.9984 \\
$80 \times 80$ & 0.0028 & 1.0000 & $0.0327 \times 10^{-3}$ & 1.9996 \\
\hline
\end{tabular}

Table 3 Numerical results at $t=0.75$ with $\tau=5 h$

\begin{tabular}{lllll}
\hline$m \times m$ & $\left\|u^{n}-U_{h}^{n}\right\|_{1, h}$ & Order & $\left\|U_{h}^{n}-\operatorname{lh}_{h} u^{n}\right\|_{1, h}$ & Order \\
\hline $20 \times 20$ & 0.0144 & - & $0.6725 \times 10^{-3}$ & - \\
$40 \times 40$ & 0.0072 & 0.9999 & $0.1679 \times 10^{-3}$ & 2.0021 \\
$80 \times 80$ & 0.0036 & 1.0000 & $0.0420 \times 10^{-3}$ & 1.9996 \\
\hline
\end{tabular}

Table 4 Numerical results at $t=1.0$ with $\tau=5 h$

\begin{tabular}{lllll}
\hline$m \times m$ & $\left\|u^{n}-U_{h}^{n}\right\|_{1, h}$ & Order & $\left\|U_{h}^{n}-I_{h} u^{n}\right\|_{1, h}$ & Order \\
\hline $20 \times 20$ & 0.0185 & - & $0.8608 \times 10^{-3}$ & - \\
$40 \times 40$ & 0.0092 & 0.9999 & $0.2154 \times 10^{-3}$ & 1.9988 \\
$80 \times 80$ & 0.0046 & 1.0000 & $0.0539 \times 10^{-3}$ & 1.9997 \\
\hline
\end{tabular}

Table 5 Numerical results at $t=0.25$ with $\tau=5 h$

\begin{tabular}{lllll}
\hline$m \times m$ & $\left\|\boldsymbol{p}^{n}-\boldsymbol{P}_{h}^{n}\right\|_{0}$ & Order & $\left\|\boldsymbol{\Pi}_{h} \boldsymbol{p}^{n}-\boldsymbol{P}_{h}^{n}\right\|_{0}$ & Order \\
\hline $20 \times 20$ & 0.0087 & - & $0.3948 \times 10^{-3}$ & - \\
$40 \times 40$ & 0.0044 & 0.9998 & $0.0988 \times 10^{-3}$ & 1.9980 \\
$80 \times 80$ & 0.0022 & 0.9999 & $0.0247 \times 10^{-3}$ & 1.9995 \\
\hline
\end{tabular}


Table 6 Numerical results at $t=0.5$ with $\tau=5 h$

\begin{tabular}{lllll}
\hline$m \times m$ & $\left\|\boldsymbol{p}^{n}-\boldsymbol{P}_{h}^{n}\right\|_{0}$ & Order & $\left\|\boldsymbol{\Pi}_{h} \boldsymbol{p}^{n}-\boldsymbol{P}_{h}^{n}\right\|_{0}$ & Order \\
\hline $20 \times 20$ & 0.0112 & - & $0.5102 \times 10^{-3}$ & - \\
$40 \times 40$ & 0.0056 & 0.9998 & $0.1277 \times 10^{-3}$ & 1.9980 \\
$80 \times 80$ & 0.0028 & 0.9999 & $0.0319 \times 10^{-3}$ & 1.9995 \\
\hline
\end{tabular}

Table 7 Numerical results at $t=0.75$ with $\tau=5 h$

\begin{tabular}{lllll}
\hline$m \times m$ & $\left\|\boldsymbol{p}^{n}-\boldsymbol{P}_{h}^{n}\right\|_{0}$ & Order & $\left\|\boldsymbol{\Pi}_{h} \boldsymbol{p}^{n}-\boldsymbol{P}_{h}^{n}\right\|_{0}$ & Order \\
\hline $20 \times 20$ & 0.0144 & - & $0.6548 \times 10^{-3}$ & - \\
$40 \times 40$ & 0.0072 & 0.9998 & $0.1639 \times 10^{-3}$ & 1.9980 \\
$80 \times 80$ & 0.0036 & 0.9999 & $0.0410 \times 10^{-3}$ & 1.9995 \\
\hline
\end{tabular}

Table 8 Numerical results at $t=1.0$ with $\tau=5 h$

\begin{tabular}{lllll}
\hline$m \times m$ & $\left\|\boldsymbol{p}^{n}-\boldsymbol{P}_{h}^{n}\right\|_{0}$ & Order & $\left\|\boldsymbol{\Pi}_{h} \boldsymbol{p}^{n}-\boldsymbol{P}_{h}^{n}\right\|_{0}$ & Order \\
\hline $20 \times 20$ & 0.0185 & - & $0.8402 \times 10^{-3}$ & - \\
$40 \times 40$ & 0.0092 & 0.9998 & $0.2103 \times 10^{-3}$ & 1.9980 \\
$80 \times 80$ & 0.0046 & 0.9999 & $0.0526 \times 10^{-3}$ & 1.9995 \\
\hline
\end{tabular}

Table 9 Convergence results of $\left\|U_{h}^{n}-\ln u^{n}\right\|_{1, h}$ with $h=\frac{1}{128}$ and $\tau=k h$

\begin{tabular}{lllll}
\hline$t$ & $k=1$ & $k=4$ & $k=8$ & $k=16$ \\
\hline$t=0.25$ & $8.2423 \times 10^{-6}$ & $1.7780 \times 10^{-5}$ & $1.0060 \times 10^{-4}$ & $4.3915 \times 10^{-4}$ \\
$t=1$ & $1.7553 \times 10^{-6}$ & $3.7559 \times 10^{-5}$ & $2.1234 \times 10^{-4}$ & $9.0638 \times 10^{-4}$ \\
\hline
\end{tabular}

Table 10 Convergence results of $\left\|U_{h}^{n}-\ln u^{n}\right\|_{1, h}$ with $h=\frac{1}{128}$ and $\tau=k h$

\begin{tabular}{lllll}
\hline$t$ & $k=1$ & $k=4$ & $k=8$ & $k=16$ \\
\hline$t=0.25$ & $8.2563 \times 10^{-6}$ & $1.7347 \times 10^{-5}$ & $9.8167 \times 10^{-5}$ & $4.2847 \times 10^{-4}$ \\
$t=1$ & $1.7147 \times 10^{-5}$ & $3.6645 \times 10^{-5}$ & $2.0720 \times 10^{-4}$ & $8.8446 \times 10^{-4}$ \\
\hline
\end{tabular}

To show the unconditional stability, we test the linearized MFEM, $h=\frac{1}{128}$, and the large time steps $\tau=h, 4 h, 8 h$, and $16 h$, respectively. We present the numerical results in Tables 9 and 10, which suggest that the scheme is stable for large time steps.

\section{Conclusion}

In this paper, we have established unconditional error estimates for a low order nonconforming MFEMs of a two-dimensional nonlinear reaction-diffusion equation. A striking feature of our analysis is that we control the $L^{p}$ norm of $\varphi_{h} \in M$ by broken $H^{1}$ norm $\left\|\varphi_{h}\right\|_{1, h}$, which leads to the superclose and superconvergence unconditionally with nonconforming MFEM. Some new splitting techniques for the consistency error terms and nonlinear terms may play a crucial role in the error estimates. It should be noted that extension to a three-dimensional nonlinear reaction-diffusion equation can also be obtained with slight change of notations. The techniques developed in this work can be used to analyze MFEMs of more general nonlinear parabolic systems.

Acknowledgements

The authors would like to thank the referees for their valuable suggestions which helped to improve this work. 
Abbreviations

MFEM, mixed finite element method; MFE, mixed finite element; PDEs, partial differential equations.

\section{Availability of data and materials}

The data are numerical results, please contact author for data requests.

\section{Competing interests}

The authors declare that they have no competing interests.

\section{Authors' contributions}

$\mathrm{HZ}$ carried out theoretical calculation, participated in the design of the study, and drafted the manuscript.

AW participated in its design and helped to draft the manuscript. All authors read and approved the final manuscript.

\section{Publisher's Note}

Springer Nature remains neutral with regard to jurisdictional claims in published maps and institutional affiliations.

Received: 9 June 2018 Accepted: 5 November 2018 Published online: 14 November 2018

\section{References}

1. Basse, B., Baguley, B., Marshall, E., et al.: A mathematical model for analysis of the cell cycle in cell lines derived from human tumors. J. Math. Biol. 47(4), 295-312 (2003)

2. Wang, X., Li, Z.: Dynamics for a type of general reaction-diffusion model. Nonlinear Anal. 67(9), 2699-2711 (2007)

3. Beta, C., Bertram, M., Mikhailov, A., et al.: Controlling turbulence in a surface chemical reaction by time-delay autosynchronization. Phys. Rev. E 67(2), 274-289 (2003)

4. Fisher, R.A.: The wave of advance of advantageous genes. Annu. Eugen. 7(4), 355-369 (1937)

5. File, P.C., Mcleod, J.B.: The approach of solutions of nonlinear diffusion equations to travelling front solutions. Arch. Ration. Mech. Anal. 65(4), 335-361 (1977)

6. Sherratt, J.A., Marchant, B.P.: Algebraic decay and variable speeds in wavefront solutions of a scalar reaction-diffusion equation. IMA J. Appl. Math. 56(3), 289-302 (1996)

7. Ramos, J.I.: Linearization methods for reaction-diffusion equations: multidimensional problems. Appl. Math. Comput. 88(2), 225-254 (1997)

8. Ramos, J.I.: Iterative and non-iterative, full and approximate factorization methods for multidimensional reaction-diffusion equations. Appl. Math. Comput. 174(2), 1586-1608 (2006)

9. Garvie, M.R.: Finite-difference schemes for reaction-diffusion equations modeling predator-prey interactions in Matlab. Bull. Math. Biol. 69(3), 931-956 (2007)

10. Ruuth, S.: Implicit-explicit method for reaction-diffusion problem in pattern formulation. J. Math. Biol. 34(2), 148-176 (1995)

11. Madzvamuse, A.: Time-stepping schemes for moving grid finite elements applied to reaction-diffusion systems on fixed and growing domains. J. Comput. Phys. 214(1), 239-263 (2006)

12. Soane, A.M., Gobbert, M.K., Seidman, T.I.: Numerical exploration of a system of reaction-diffusion equations with internal and transient layers. Nonlinear Anal., Real World Appl. 6(5), 914-934 (2005)

13. Raviart, P.A., Thomas, J.M.: A mixed finite element method for second order elliptic problems. In: Mathematical Aspects of the Finite Element Method. Lecture Notes in Math., vol. 606, pp. 292-315. Springer, New York (1977)

14. Dawson, C., Sun, S., Wheeler, M.: Compatible algorithm for coupled flow and transport. Comput. Methods Appl. Mech. Eng. 193(23), 2562-2580 (2004)

15. Chen, L., Chen, Y.: Two-grid method for nonlinear reaction-diffusion equations by mixed finite element methods. J. Sci. Comput. 49(3), 383-401 (2011)

16. Garcia, M.: Improved error estimates for mixed finite element approximations for nonlinear parabolic equations: the continuously-time case. Numer. Methods Partial Differ. Equ. 10, 129-149 (1994)

17. Garcia, M.: Improved error estimates for mixed finite element approximations for nonlinear parabolic equations: the discrete-time case. Numer. Methods Partial Differ. Equ. 10(2), 149-169 (2010)

18. Li, W., Allen, M.B.: Two grid methods for mixed finite element solution of coupled reaction-diffusion systems. Numer. Methods Partial Differ. Equ. 15(5), 589-604 (2015)

19. Johnson, C., Thomee, V.: Error estimates for some mixed finite element methods for parabolic type problems. RAIRO. Anal. Numér. 15(1), 41-78 (2009)

20. Kim, D., Park, E., Seo, B.: Two-scale product approximation for semilinear parabolic problems in mixed methods. J. Korean Math. Soc. 51(2), 267-288 (2014)

21. Wu, L., Allen, M.: A two-grid method for mixed finite-element solution of reaction-diffusion equations. Numer. Methods Partial Differ. Equ. 15(3), 317-332 (2015)

22. Chen, Y., Liu, H., Liu, S.: Analysis of two-grid methods for reaction-diffusion equations by expanded mixed finite element methods. Int. J. Numer. Methods Eng. 69(2), 408-422 (2010)

23. Chen, Y., Huang, Y., Yu, D.: A two-grid method for expanded mixed finite-element solution of semilinear reaction-diffusion equations. Int. J. Numer. Methods Eng. 57(2), 193-209 (2003)

24. Thomée, V.: In: Galerkin Finite Element Methods for Parabolic Problems. Springer Series in Computational Mathematics, Sweden (2000)

25. Li, B., Sun, W.: Error analysis of linearized semi-implicit Galerkin finite element methods for nonlinear parabolic equations. Int. J. Numer. Anal. Model. 10(3), 622-633 (2013)

26. Li, B., Sun, W.: Unconditional convergence and optimal error estimates of a Galerkin-mixed FEM for incompressible miscible flow in porous media. SIAM J. Numer. Anal. 51(4), 1959-1977 (2013)

27. Hou, Y., Li, B., Sun, W.: Error estimates of splitting Galerkin methods for heat and sweat transport in textile materials. SIAM J. Numer. Anal. 51(1), 88-111 (2013) 
28. Gao, H.: Optimal error analysis of Galerkin FEMs for nonlinear Joule heating equations. J. Sci. Comput. 58(3), 627-647 (2014)

29. Li, B., Gao, H., Sun, W.: Unconditionally optimal error estimates of a Crank-Nicolson Galerkin method for the nonlinear thermistor equations. SIAM J. Numer. Anal. 52(2), 933-954 (2014)

30. Wang, J.: A new error analysis of Crank-Nicolson Galerkin FEMs for a generalized nonlinear Schrödinger equation. J. Sci. Comput. 60(2), 390-407 (2014)

31. Shi, D., Wang, J.: Unconditional superconvergence analysis of a Crank-Nicolson Galerkin FEM for nonlinear Schrödinger equation. J. Sci. Comput. 72(3), 1093-1118 (2017)

32. Shi, D., Wang, J., Yan, F.: Unconditional superconvergence analysis for nonlinear parabolic equation with $E Q_{1}^{\text {rot }}$ nonconforming finite element. J. Sci. Comput. 70(1), 85-111 (2017)

33. Shi, D., Wang, J.: Unconditional superconvergence analysis of conforming finite element for nonlinear parabolic equation. Appl. Math. Comput. 294, 216-226 (2017)

34. Shi, D., Wang, J.: Unconditional superconvergence analysis of a linearized Galerkin FEM for nonlinear hyperbolic equation. Comput. Math. Appl. 74(4), 634-651 (2017)

35. Shi, D., Wang, J.: Unconditional superconvergence analysis for nonlinear hyperbolic equation with nonconforming finite element. Comput. Math. Appl. 305, 1-16 (2017)

36. Chen, S., Chen, H.: New mixed element schemes for second order elliptic problem. Math. Numer. Sin. 32(2), 213-218 (2010)

37. Shi, F., Yu, J., Li, K.: A new mixed finite element scheme for elliptic equations. J. Eng. Math. 28(2), 231-237 (2011)

38. Gao, H., Qiu, W.: Error analysis of mixed finite element methods for nonlinear parabolic equations. J. Sci. Comput. 77(3), 1660-1678 (2018)

39. Shi, D., Zhang, Y.: High accuracy analysis of a new nonconforming mixed finite element scheme for Sobolev equations. Appl. Math. Comput. 218(7), 3176-3186 (2011)

40. Lin, Q., Lin, J.: Finite Element Methods: Accuracy and Improvement. Beijing Science Press, Beijing (2006)

41. Lin, Q., Tobiska, L., Zhou, A.: Superconvergence and extrapolation of nonconforming low order finite elements applied to the Poisson equation. IMA J. Numer. Anal. 25(1), 160-181 (2005)

42. Shi, D., Mao, S., Chen, S.: An anisotropic nonconforming finite element with some superconvergence results. J. Comput. Math. 23(3), 261-274 (2005)

43. Shi, D., Ren, J.: Nonconforming mixed finite element approximation to the stationary Navier-Stokes equations on anisotropic meshes. Nonlinear Anal. 71(9), 3842-3852 (2009)

\section{Submit your manuscript to a SpringerOpen ${ }^{\circ}$ journal and benefit from:}

- Convenient online submission

- Rigorous peer review

- Open access: articles freely available online

- High visibility within the field

- Retaining the copyright to your article

Submit your next manuscript at $\gg$ springeropen.com 